\title{
Sequential Fluorescent Labeling Observation of Maxillary Sinus Augmentation by a Tissue-engineered Bone Complex in Canine Model
}

\author{
Xin-quan Jianga, Shao-yi Wanga, Jun Zhao, Xiu-li Zhang, Zhi-yuan Zhang* \\ Shanghai Research Institute of Stomatology, Shanghai Key Laboratory of Stomatolgoy, Shanghai Ninth People's Hospital \\ affiliated to Shanghai Jiao Tong University School of Medicine, Shanghai, China
}

\begin{abstract}
Xin-quan Jiang, Shao-yi Wang, Jun Zhao, Xiu-li Zhang, Zhiyuan Zhang. Sequential Fluorescent Labeling Observation of Maxillary Sinus Augmentation by a Tissue-engineered Bone Complex in Canine Model. International Journal of Oral Science, 1(1): 39-46, 2009
\end{abstract}

Aim To evaluate the effects of maxillary sinus floor elevation by a tissue-engineered bone complex of $\beta$-tricalcium phosphate $(\beta-\mathrm{TCP})$ and autologous osteoblasts in dogs.

Methodology Autologous osteoblasts from adult Beagle dogs were cultured in vitro. They were further combined with $\beta$-TCP to construct the tissue-engineered bone complex. 12 cases of maxillary sinus floor elevation surgery were made bilaterally in 6 animals and randomly repaired with the following 3 groups of materials: Group A (osteoblasts/ $\beta$-TCP); Group B ( $\beta$-TCP); Group C (autogenous bone) ( $n=4$ per group). A polychrome sequential fluorescent labeling was performed post-operatively and the animals were sacrificed 24 weeks after operation for
CLC number: R783.5

\section{Introduction}

As the bony height from the alveolar crest to the sinus floor is usually not adequate enough for dental implantation due to sinus pneumatization or alveolar resorption, the lack of initial stability at edentulous sites would usually lead to the failure of osseointegrated implants (Tiwana et al., 2006; Browaeys et al., 2007). As a consequence, the augmentation of the deficient osseous ridge has become an integral part of therapeutic procedures in pre-prosthetic, pre-implantology surgery (Woj- histological observation.

Results Our results showed that autologous osteoblasts were successfully expanded and the osteoblastic phenoltypes were confirmed by ALP and Alizarin red staining. The cells could attach and proliferate well on the surface of the $\beta$-TCP scaffold. The fluorescent and histological observation showed that the tissue-engineered bone complex had an earlier mineralization and more bone formation inside the scaffold than $\beta$-TCP along or even autologous bone. It had also maximally maintained the elevated sinus height than both control groups.

Conclusion Porous $\beta$-TCP has served as a good scaffold for autologous osteoblasts seeding. The tissue-engineered bone complex with $\beta$-TCP and autologous osteoblasts might be a better alternative to autologous bone for the clinical edentulous maxillary sinus augmentation.

Keywords maxillary sinus augmentation, tissue engineering, $\beta$-tricalcium phosphate, osteoblasts, fluorescent labeling towicz et al., 2007).

Among the various techniques used to regain the height of resorbed maxilla, maxillary sinus floor elevation is regarded as an effective way to restore the upper jaw (Jensen et al., 1998). The use of autogenous bone grafts in sinus augmentation has been considered as "gold standard" since its introduction (Boyne and James, 1980) because of their excellent survival for loaded implants and the degree of functionality they can afford. However, when harvesting autologous bone, side effects including donor-side morbidity, infection, pain and 
blood loss were reported (Ueda et al., 2001). Allografts and xenografts are susceptible to immunoreaction and carry the disease transmission risks. Some synthetic materials, on the contrary, have limited potential for osteoconduction, as well as osteoinduction (Sun et al., 2008).

Due to the disadvantage of the above methods, other alternative therapies need to be explored for maxillary sinus augmentation. Recent technological advances have allowed dentists to explore regeneration of bone by using novel tissue engineering techniques (Hollinger et al., 2000) where biomaterials and cells are among the key elements for the principles (Langer and Vacanti, 1993). Though there have been few animal studies or clinical case reports applied in maxillary sinus augmentation with this method, these studies only gave some preliminary evaluation of the effects (Schimming and Schmelzeisen, 2004; Beaumont et al., 2008). In this study, we have used porous $\beta$-TCP granules for the first time to combine with autologous osteoblasts to augment the maxillary sinus floor in a canine model, and a sequential fluorescent labeling observation was taken to evaluate the outcome used by this tissue-engineered bone complex.

\section{Methods and materials}

\section{Animals}

A total of 6 adult beagle dogs in healthy condition, 18 months of age with an average weight of $12.5 \mathrm{~kg}$ were used in this study. The experimental protocol was approved by the Animal Care and Experiment Committee of Ninth Peoples Hospital affiliated to Shanghai JiaoTong University, School of Medicine.

\section{Cell culture}

Under general anesthesia with 5\% sodium pentobarbital $(0.5 \mathrm{~mL} / \mathrm{kg})$, a bony sheet biopsy was obtained from the lateral cortex of the mandibular body in the apical region of the second molar area by an intraoral buccal approach, and placed immediately into phosphate buffered saline (PBS) containing of 100 units $/ \mathrm{mL}$ penicillin and $100 \mathrm{mg} / \mathrm{mL}$ streptomycin. After washing thoroughly by phosphate buffered saline (PBS) to remove blood components, the samples were cut into small fragments and cultured in $100 \mathrm{~mm}$ dishes (Corning, USA) with standard DMEM : F12 $(1: 1)$ (Gibco BRL, USA) culture medium containing $10 \%(V / V)$ fetal bovine serum (FBS; Hyclone, USA), 100 units $/ \mathrm{mL}$ penicillin and $100 \mathrm{mg} / \mathrm{mL}$ streptomycin. Explants were incubated under 95\% humidity, $5 \% \mathrm{CO}_{2}$, $37^{\circ} \mathrm{C}$ which were subsequently fed continuously by replacing the medium every 3-4 days until cell density reached $70 \%-80 \%$ confluence. Then cells were detached with $0.25 \%$ trypsin/EDTA, subcultured at a density of $1 \times 10^{5}$ cells $/ \mathrm{cm}^{2}$ in $100 \mathrm{~mm}$ dishes. The cells at passage $2-3$ were used in our study.

\section{Alkaline phosphatase staining and alizarin red $S$ calcium nodules staining}

After culturing for another 14 days, the expanded cells were measured by alkaline phosphatase (ALP) staining and alizarin red $\mathrm{S}$ staining. Briefly, the cells were fixed for 10 minutes at $4^{\circ} \mathrm{C}$ and incubated with a mixture of naphthol AS-MX phosphate, N,N-dimethylformamide and fast blue BB salt (ALP kit, Hongqiao, China)(Jiang et al., 2006). The calcium nodules staining method consisted of the cells being cultured with $10 \mathrm{mg} / \mathrm{L}$ alizarin red S (Sigma Aldrich, USA) for 5-7 days before observed using a fluorescent microscope (Leica DM 1RB, Germany).

\section{Preparation of Osteoblasts/ $\beta$-TCP construct}

$\beta$-TCP granules (Shanghai Bio-Lu Biomaterials Co.,Ltd., China; diameter: $1.5-2.5 \mathrm{~mm}$;) were sterilized by ${ }^{60} \mathrm{Co}$ irradiation before use. For cell seeding, osteoblasts were detached from culture dishes, centrifuged to remove supernatant, and then resuspended in the culture media without FBS at a density of $2 \times 10^{7}$ cells $/ \mathrm{mL}$. Cells in suspension were slowly combined with the $\beta$-TCP granules till a final saturation. After being incubated for additional 4 hours to allow cell's initial attachment, implantation surgeries was performed in vivo.

In a parallel experiment, $3 \mathrm{~mm} \times 3 \mathrm{~mm} \times 3 \mathrm{~mm}$ cuboids were prepared and seeded with osteoblasts at an identical cell density. 1 day after, the 
constructs were fixed in $2 \%$ glutaric dialdehyde for 2 hours, cut into two halves, and then subjected for scanning electron microscopy examination (Philips SEM XL-30, Netherlands).

\section{Surgical procedure}

Under general anesthesia through intramuscular injection of ketamine $(10 \mathrm{mg} / \mathrm{kg})$ all dogs were prepared by extraction of maxillary third premolar and first molar bilaterally 8 weeks before the maxillary sinus augmentation surgery, and gingival was closed after the operation. 12 alveolar augmentation surgeries in 6 animals were made bilaterally and randomly repaired with the following 3 groups of grafts: Group A consisted of tissue-engineered osteoblasts/ $\beta$-TCP complex ( $n=4)$; Group B consisted of $\beta$-TCP alone $(n=4)$; as a positive control, Group $\mathrm{C}$ consisted of autogenous bone obtained from iliac bone $(n=4)$.

Based on the technique described by Kent and Block (Kent and Block, 1989), bilateral maxillary sinus elevation surgeries were carried out identically. The edentulous region was opened by crestal incision. The mucoperiosteal flap was reflected on the buccal cortical plate, extending from the first maxillary premolar to the second maxillary molar. A bone window of $1.5 \mathrm{~cm} \times 2 \mathrm{~cm}$ was marked using round burs, After removal of the bone, the sinus membrane was elevated from the maxillary sinus floor, taking care to avoid perforation of the antral membrane. After creating the extrasinusoidal space, and around $1.2 \mathrm{~mL}$ tissue-engineered bone substitutes or autologous bone were inserted to elevate the sinus to reach an average height of $10 \mathrm{~mm}$ to the floor (Figure 1). Finally, the mucoperiosteal flap was repositioned and sutured.

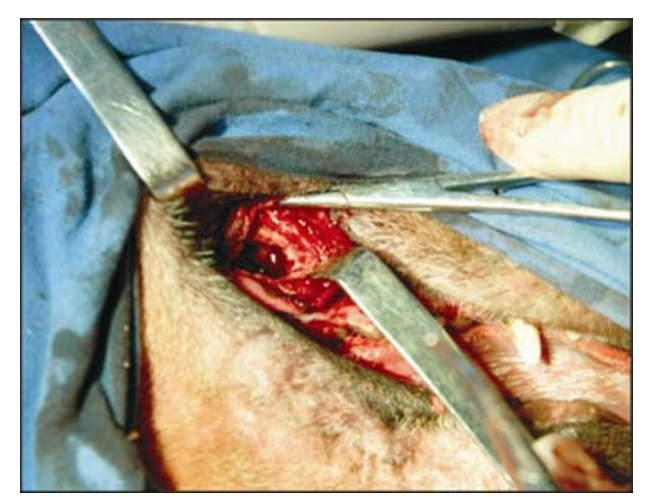

Figure 1 Maxillary sinus augmentation surgery
For the autogenous bone graft, an incision of $5 \mathrm{~cm}$ was made and a corticocancellus bone block was harvested from the anterior iliac crest. The periosteum and skin flap were replaced and sutured. The corticocancellous bone block was ground with a bone mill (Leibinger, Germany) and the bone graft was stored in a physiologic saline solution before it was grafted to the maxillary sinus.

\section{Sequential fluorescent labeling}

A polychrome sequential fluorescent labeling method was carried out to label the mineralized tissue and assess the time course of new bone formation and mineralization. At 4, 12, 20 weeks after the operation, the animals were intraperitoneally administered with $25 \mathrm{mg} / \mathrm{kg}$ hydrochloride tetracycline (TE) (Sigma Aldrich, USA), $20 \mathrm{mg} / \mathrm{kg}$ calcein (CA) (Sigma Aldrich, USA), and $30 \mathrm{mg} / \mathrm{kg}$ alizarin red S (AL) (Sigma Aldrich, USA) respectively.

\section{Sample preparation, the height as well as histological and fluorescent analysis}

The dogs were sacrificed at 24 weeks after surgery. The augmented maxillary sinus was fixed in $10 \%$ buffered formalin (pH 7.4). One half cut along the perpendicular plane was dehydrated in ascending concentrations of alcohol from $75 \%$ to $100 \%$, and finally embedded in polymethymetacrylate (PMMA). The specimens were cut in $150 \mu \mathrm{m}$ thick sections using a microtome (Leica, Germany), and were subsequently ground and polished to a final thickness of about $40 \mu \mathrm{m}$.

The measurements of elevated height were performed on gross cross section. It was defined as the distance between the bottom and the top of augmented maxillary sinus on undecalcified specimens, and the mean value of four specimen was used to calculate the height of each group.

Undecalcified sections were observed for fluorescent labeling under confocal laser scanning microscope (CLSM) (Leica TCS Sp2 AOBS, Germany), excitation/emission wavelengths for each of the fluorescence was used as $405 \mathrm{~nm} / 580 \mathrm{~nm}$ (TE), $488 \mathrm{~nm} / 517 \mathrm{~nm}(\mathrm{CA}), 543 \mathrm{~nm} / 617 \mathrm{~nm}(\mathrm{AL})$, respectively. Then the sections were stained with 

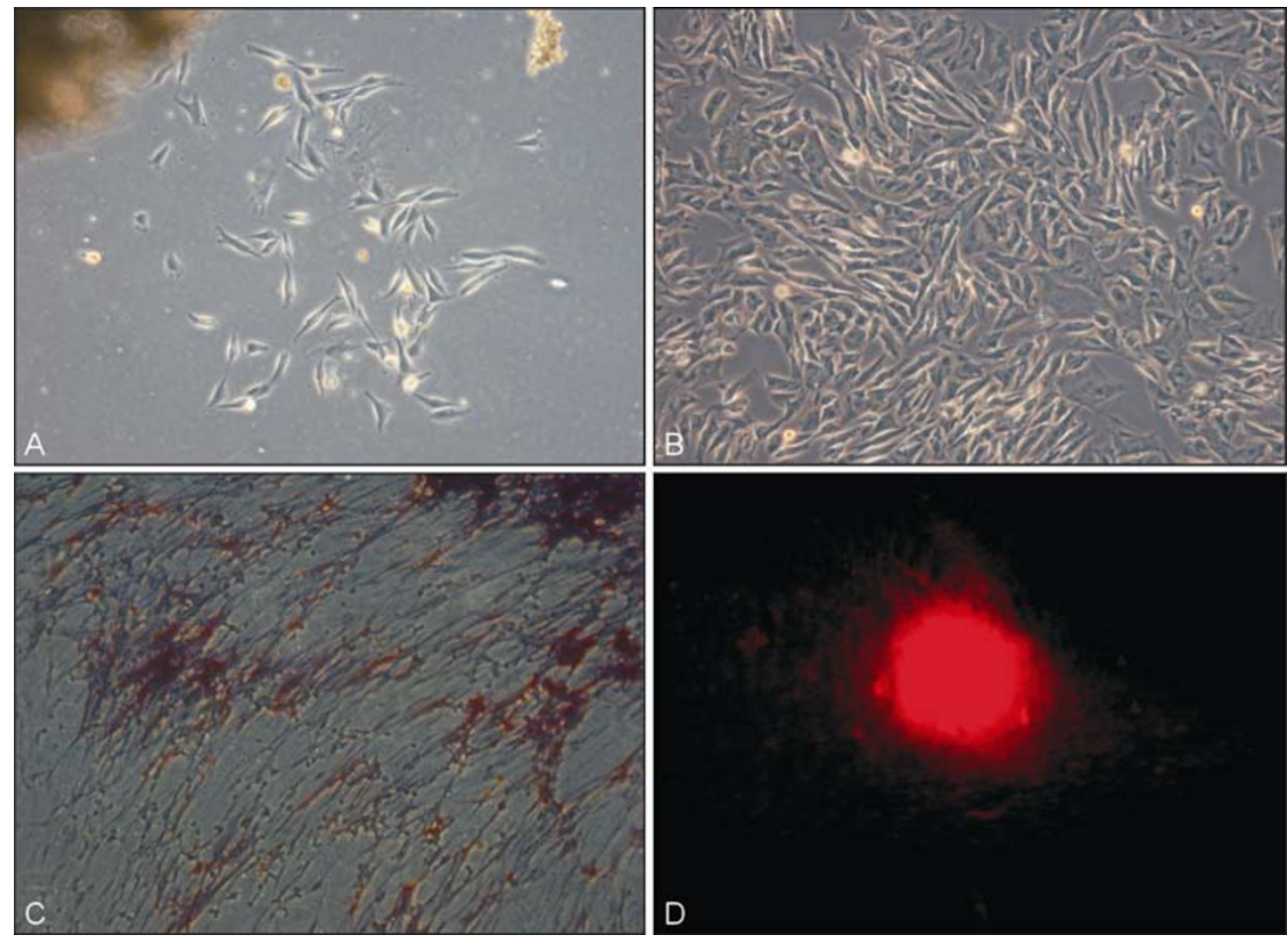

Figure 2 Cell culture and osteoblastic phenotype tests

(A): Cells were found growing around bone fragments after initial incubation $(100 \times)$. (B): Cells proliferated quickly afterwards to reach a confluence $(100 \times) .(C)$ : Alkaline phosphatase-positive staining area $(50 \times)$. (D): Mineralized calcium nodules shown by alizarin red $\mathrm{S}$ staining $(100 \times)$.

van Gieson's picro fuchsin for histologic observation.

\section{Statistical analysis}

Statistically significant differences $(P<0.05)$ between the various groups were measured using ANOVA and SNK post hoc. All statistical analysis was carried out using a SAS 6.12 statistical software package (Cary, USA).

\section{Results}

\section{Cell culture and osteoblastic phenotype tests}

Cells were found growing around bone fragments 5-9 days after initial incubation (Figure 2A), and proliferated quickly afterwards to reach a confluence after approximately another 4-7 days (Figure 2B) for subculture. Around 14 days after seeding for expanded cells, ALP-positive staining (Figure 2C) and mineralized calcium nodules alizarin red S staining (Figure 2D) were observed in cultured cells, demonstrating that those cultured cells maintained the osteblastic phenotype after sufficient in vitro expanding.

\section{Adhesion and spreading of osteoblasts on the material}

Scanning electron microscope was used to determine the pores of $\beta$-TCP with a dimension of $450 \mu \mathrm{m}$ and the interconnection pores diameter of $(150 \pm 50) \mu \mathrm{m}$ (Figure 3A). 1 day after the osteoblasts were combined with the material, cells attached to and spread on the surface of the scaffold in vitro (Figure 3B). The results suggested that the material was a suitable biomaterial as it facilitated osteoblasts initial adhesion and spreading onto its surface.

\section{The height of augmented maxillary sinus and histological analysis}

To evaluate the effects of augmented maxillary 


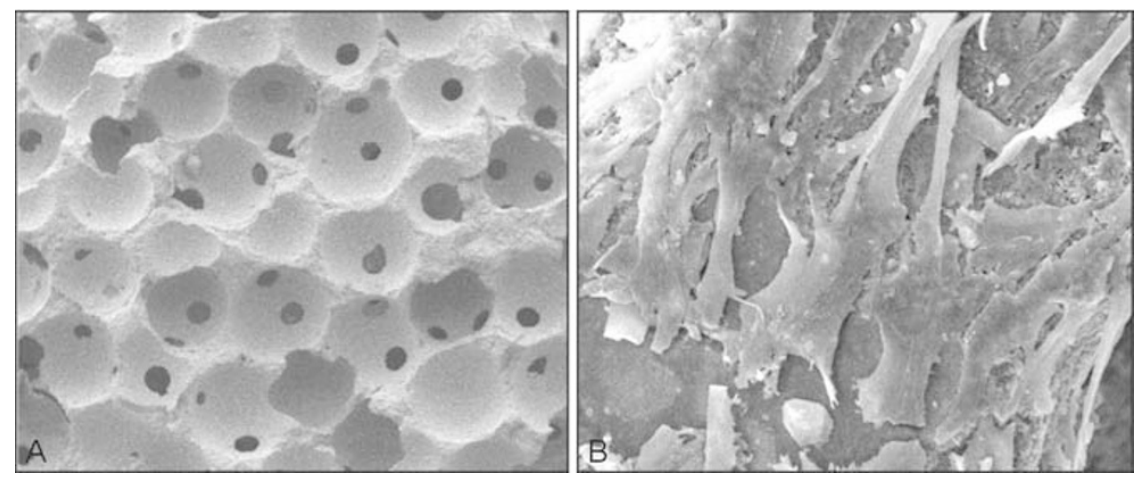

Figure 3 Scanning electron microscopic evaluation of the scaffold microstructure, and osteoblasts attaching to and spreading on the surface of the scaffold

(A): Scanning electron microscopic evaluation of the scaffold microstructure (50×). (B): 1 day after the osteoblasts were combined with $\beta$-TCP, they could be seen attaching and spreading on the surface of the scaffold $(600 \times)$.

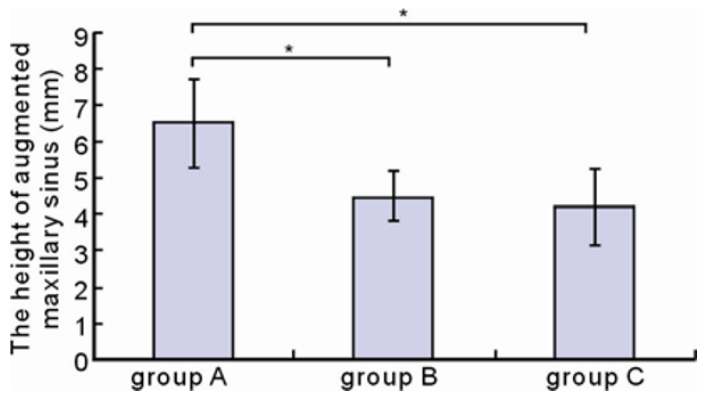

Figure 4 The height of augmented maxillary sinus analysis

There is significant difference between Group A and Group B or Group C $(P<0.05)$.

(*indicates significant differences $P<0.05$ ).

sinus, the height was measured. As Figure 4 showed, the height of tissue-engineered maxillary sinus augmentation remained with $(6.55 \pm 1.21) \mathrm{mm}$ at 24 weeks, by comparison, the augmented height decreased significantly to only $(4.51 \pm 0.69) \mathrm{mm}$ for group B ( $\beta$ - TCP control), which is comparable to the autogenous bone group $(4.26 \pm 1.03) \mathrm{mm}(P>$ $0.05)$. Both Group B and Group $C$ were significant lower than group A $(P<0.05)$. Under light microscopy (Leica DM 2500, Germany), the specimens in group A demonstrated a significant amount of new bone formation even at center portion of the block, which is obviously higher than that of group $\mathrm{B}$ or group $\mathrm{C}$ (Figure 5A, 5B, 5C).

\section{Fluorochrome microscopy}

The deposition of mineralized bone matrix was observed in Group A, Group B and Group C for different time points as demonstrated by tetracycline (yellow), calcein (green) and alizarin red $\mathrm{S}$ (red). At week 4, fluorescent-labeling areas in Group A were larger than those in Group B or Group C, as evidenced by the fluorescence of tetracycline (yellow). There was no significant difference among the three Groups detected by calcein (green) fluorescent labeling at week 12, however, 20 weeks later, the mineralization of new bone was present more frequently in Group $\mathrm{C}$ than in Group A or Group B according to alizarin red S labeling. These data suggested that seeding osteoblasts contributed to the enhanced mineralized area at an earlier stage for the tissue-engineered group. While in Group C, the absorption of autogenous illum bone was probably predominant at earlier stage, since obvious new bone regeneration and mineralization could be seen till at a later stage of week 20 (Figrue 6A, 6B, 6C).

\section{Discussion}

Sinus augmentation has become a standard procedure to increase bony height in the posterior maxilla. Though several procedures and materials for augmenting bone height have been reported (Browaeys et al., 2007; Knabe et al., 2008; Yamada et al., 2008), there was still a lack of objective scientific data regarding the beneficial effects about using tissue-engineered bone complex for augmention of maxillary sinus. In this study, we 


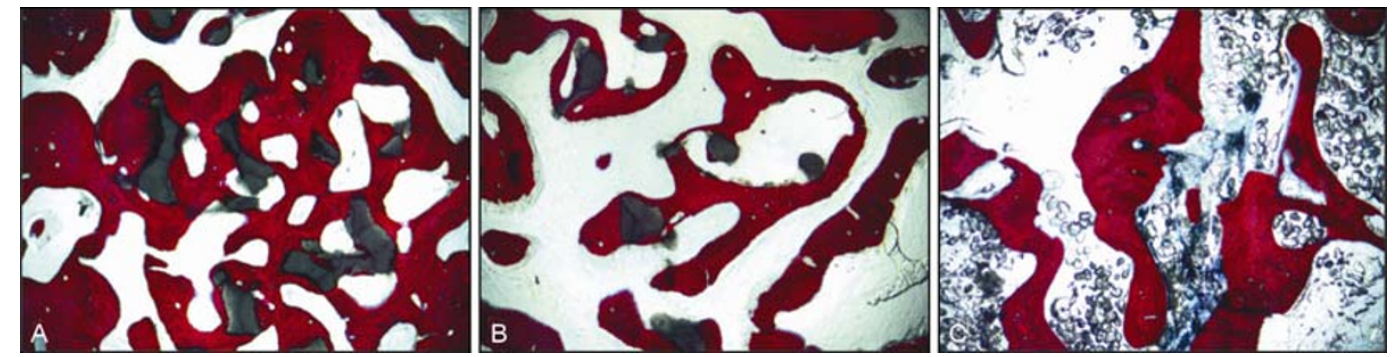

Figure 5 The photomicrograph of bone formation inside augmented maxillary sinus for different groups $(40 \times)$
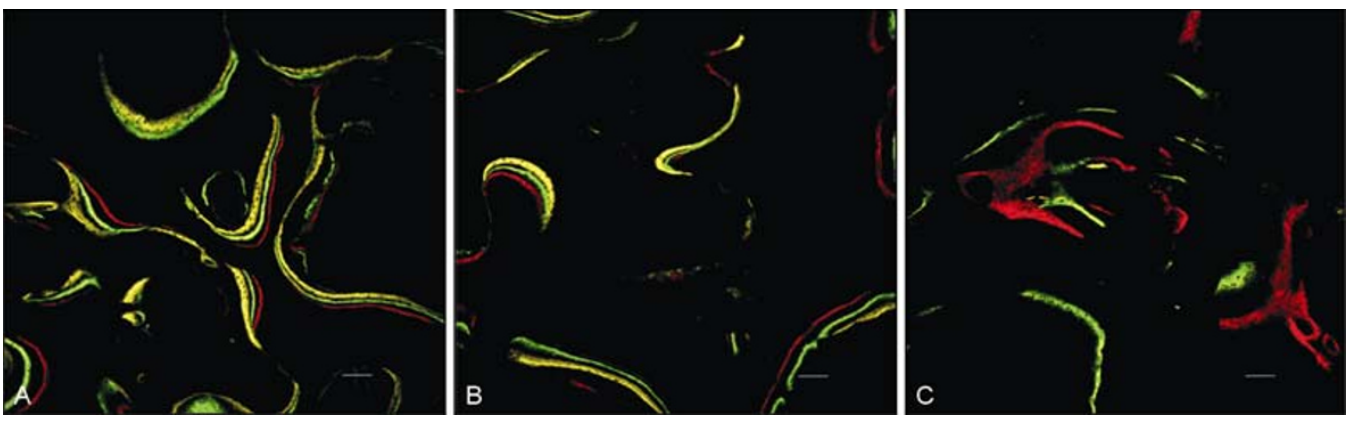

Figure 6 New bone formation and mineralization were determined by fluorescent observation the mineralization level for different groups

Determined by TE, CA and AL fluorescent observation the mineralization level at 4, 12, and 20 weeks after operation for different groups (scale bar $100 \mu \mathrm{m}$ ).

demonstrated that tissue-engineered bone with autologous osteoasts and a biodegradable $\beta$-TCP scaffold achieved an earlier bone formation and mineralization and maximally maintained the elevated space of the maxillary sinus when compared with $\beta$-TCP scaffold alone or even autogenous bone, which may facilitate to ensure the placement of dental implants with sufficient length and satisfy initial stability.

Autologous osteoblasts derived from periosteum or bone, has been successfully used previously to regenerate bones in animal experiments, or in clinic (Rai et al., 2004; Strietzel, 2006). They are easy to harvest, and compared with mixed properties of bone marrow stromal cells, they are relatively pure, and have inherent bone formation capability (Hu et al., 2003; Lee et al., 2006). We have been able to isolate the osteoblasts from a small sheet of cortical bone in mandible through an intraoral surgical procedure. We were able to obtain enough cells in 3 passages with definite osteoblastic phenotypes, which was sufficient for constructs to fill a void volume of as large as $3 \mathrm{~cm}^{3}$. Such a surgical biopsy is minimally invasive, and convenient for oral surgeons.
The $\beta$-TCP we selected has good biocompatibility and osteo-conductive capacity. Compared with other bone substitutes (e.g. collagen scaffolds), $\beta$-TCP is characterized by its precisely defined physical and chemo-crystalline properties, high level of purity and uniformity of chemical composition, so that its biological reactions can be predicted reliably (Horch et al., 2006). It can be fabricated into high porosity scaffolds with good interconnectivity, which will ensure intercellular communication among osteogenic cells rested in lacunae. The macro-porosity of the material will facilitate cells adhesion and growth, and facilitate bony ingrowth and especially vascularization (Dong et al., 2002). It has been used in oral and maxillofacial surgery such as sinus floor augmentation and repair of deficient alveolar bone at immediate implant (Zerbo et al., 2001; Ormianer et al., 2006). However, its potential as a tissue-engineered scaffold is not well established for maxillary sinus augmentation.

A polychrome fluorescent labeling observation in the current animal study showed that new bone formation and mineralization in Group A (osteoblasts/ $\beta$-TCP) was more than Group B ( $\beta$-TCP) or 
Group C (autologous bone) at 4 weeks according to the area of TE labeling (yellow). There were no significant difference were detected among three groups at 12 weeks according to CA labeling (green). While 20 weeks after operation, AL labeling (red) was more obviously observed in Group C. The data indicated that, in Group A, seeding osteoblasts with osteoconductive $\beta$-TCP, could promote new bone formation and mineralization inside the elevated space at a much earlier stage, while for $\beta$-TCP scaffold control, the osteoconductive $\beta$-TCP alone can be used to achieve only a delayed and less effective mineralization in augmented maxillary sinus. Unexpectedly, for autogenuous illum bone, resorption of grafts seemed to be the dominant phenomena with only few new bone deposition and mineralization in maxillary sinus being observed at an earlier period, which increased till at a later stage of 20 weeks.

Coincided with the mineralization observation, the histological observation of new bone formation at 24 weeks after operation showed that new bone area inside the tissue-engineered bone was much larger than those of the control groups. At this point in time, the augmented sinus height analysis suggested that the tissue-engineered bone could maximally maintained the augmented space. For group $\mathrm{B}, \beta$-TCP alone achieved a much less elevated space than group $A$, indicating that, without osteoblasts, $\beta$-TCP granule may be resorbed more rapidly. Interestingly, the height of the autograft also reduced significantly at 24 weeks postoperation. Similar trend on autologous bony resorption were reported in other studies (Watanabe et al., 1999; Wada et al., 2001). One explanation might be that cancellous bone in the autogenous bone graft could not withstand sinus pressures for long periods of time, and as such, start to lose their density and height during the first several weeks (Jensen et al., 1998). Some publicshed clinical data showed that the absorption rate using autogenous bone in sinus augmentation was 47\% 6-7 months after surgery (Johansson et al., 2001). In fact, the resorption of autogenous bone graft has not only been reported in a sinus lifting model but has also been described in studies that explored ridge augmentation and bone defect's restoration (Roccuzzo et al., 2007). By comparison, the tissue-engineered bone complex in the current study might have withstood the sinus air pressure and maintained the highest elevated space to achieve an increased effect than scaffold along or autologous bone.

\section{Conclusion}

In conclusion, porous $\beta$-TCP had served as a good scaffold for autologous osteoblasts seeding, and tissue engineered bone complex with $\beta$-TCP and autologous osteoblasts might be a superior alternative to autologous bone for the clinical edentulous maxillary sinus augmentation.

\section{Acknowledgements}

This work was supported by National Natural Science Foundation of China 30400502, 30772431. Science and Technology Commission of Shanghai Municipality 07DZ22007, 08410706400, 08JC141 4400, S30206, Y0203, T0202. Shanghai Risingstar Program 05QMX1426, 08QH14017. Shanghai ShuGuang 07SG19.

\section{References}

Boyne PJ, James RA (1980). Grafting of the maxillary sinus floor with autogenous marrow and bone. J Oral Surg, 38(8): 613-616.

Browaeys H, Bouvry P, De Bruyn H (2007). A literature review on biomaterials in sinus augmentation procedures. Clin Implant Dent Relat Res, 9(3): 166-177.

Dong J, Uemura T, Shirasaki Y, Tateishi T (2002). Promotion of bone formation using highly pure porous beta-TCP combined with bone marrow-derived osteoprogenitor cells. Bioma terials, 23(23): 4493-4502.

Hollinger JO, Winn S, Bonadio J (2000). Options for tissue engineering to address challenges of the aging skeleton. Tissue Eng, 6(4): 341-350.

Horch HH, Sader R, Pautke C, Neff A, Deppe H, Kolk A (2006). Synthetic, pure-phase beta-tricalcium phosphate ceramic granules (Cerasorb) for bone regeneration in the reconstructive surgery of the jaws. Int $J$ Oral Maxillofac Surg, 35(8): 708-713.

$\mathrm{Hu}$ Y, Chan E, Wang SX, Li B (2003). Activation of p38 mitogen-activated protein kinase is required for osteoblast differentiation. Endocrinology, 144(5): 2068- 2074. 
Jensen OT, Shulman LB, Block MS, Iacono VJ (1998). Report of the Sinus Consensus Conference of 1996. Int J Oral Maxillofac Implants, 13(Suppl): 11-45.

Jiang X, Gittens SA, Chang Q, Zhang X, Chen C, Zhang Z (2006). The use of tissue-engineered bone with human bone morphogenetic protein-4-modified bone-marrow stromal cells in repairing mandibular defects in rabbits. Int J Oral Maxillofac Surg, 35(12): 1133-1139.

Johansson B, Grepe A, Wannfors K, Hirsch JM (2001). A clinical study of changes in the volume of bone grafts in the atrophic maxilla. Dentomaxillofac Radiol, 30(3): 157-161.

Kent JN, Block MS (1989). Simultaneous maxillary sinus floor bone grafting and placement of hydroxylapatitecoated implants. J Oral Maxillofac Surg, 47(3): 238242.

Knabe C, Koch C, Rack A, Stiller M (2008). Effect of betatricalcium phosphate particles with varying porosity on osteogenesis after sinus floor augmentation in humans. Biomaterials, 29(14): 2249-2258.

Langer R, Vacanti JP (1993). Tissue engineering. Science, 260(5110): 920-926.

Lee MK, Lim SW, Yang H, Sung SH, Lee HS, Park MJ, et al. (2006). Osteoblast differentiation stimulating activity of biflavonoids from Cephalotaxus koreana. Bioorg Med Chem Lett, 16(11): 2850-2854.

Ormianer Z, Palti A, Shifman A (2006). Survival of immediately loaded dental implants in deficient alveolar bone sites augmented with beta-tricalcium phosphate. Implant Dent, 15(4): 395-403.

Rai B, Teoh SH, Ho KH, Hutmacher DW, Cao T, Chen F, et al. (2004). The effect of rhBMP-2 on canine osteoblasts seeded onto 3D bioactive polycaprolactone scaffolds. Biomaterials, 25(24): 5499-5506.

Roccuzzo M, Ramieri G, Bunino M, Berrone S (2007). Autogenous bone graft alone or associated with titanium mesh for vertical alveolar ridge augmentation: A controlled clinical trial. Clin Oral Implants Res, 18(3): 286-294.

Schimming R, Schmelzeisen R (2004). Tissue-engineered bone for maxillary sinus augmentation. J Oral Maxillofac Surg, 62(6): 724-729.

Strietzel FP (2006). Tissue-engineered bone for lateral alveolar ridge augmentation: A case report. Int J Oral Maxillofac Implants, 21(1): 131-135.

Sun XJ, Zhang ZY, Wang SY, Gittens SA, Jiang XQ, Chou LL (2008). Maxillary sinus floor elevation using a tissue-engineered bone complex with OsteoBone and bMSCs in rabbits. Clin Oral Implants Res, 19(8): 804813.

Tiwana PS, Kushner GM, Haug RH (2006). Maxillary sinus augmentation. Dent Clin North Am, 50(3): 409424.

Ueda M, Tohnai I, Nakai H (2001). Tissue engineering research in oral implant surgery. Artif Organs, 25(3): 164-171.

Wada K, Niimi A, Watanabe K, Sawai T, Ueda M (2001). Maxillary sinus floor augmentation in rabbits: A comparative histologic-histomorphometric study between rhBMP-2 and autogenous bone. Int $J$ Periodontics Restorative Dent, 21(3): 252-263.

Watanabe K, Niimi A, Ueda M (1999). Autogenous bone grafts in the rabbit maxillary sinus. Oral Surg Oral Med Oral Pathol Oral Radiol Endod, 88(1): 26-32.

Wojtowicz A, Chaberek S, Urbanowska E, Ostrowski K (2007). Comparison of efficiency of platelet rich plasma, hematopoieic stem cells and bone marrow in augmentation of mandibular bone defects. N Y State Dent $J, 73(2)$ : 41-45.

Yamada Y, Nakamura S, Ito K, Kohgo T, Hibi H, Nagasaka $\mathrm{T}$, et al. (2008). Injectable tissue-engineered bone using autogenous bone marrow-derived stromal cells for maxillary sinus augmentation: clinical application report from a 2-6-year follow-up. Tissue Eng Part A, 14(10): 1699-1707.

Zerbo IR, Bronckers AL, de Lange GL, van Beek GJ, Burger EH (2001). Histology of human alveolar bone regeneration with a porous tricalcium phosphate. A report of two cases. Clin Oral Implants Res, 12(4): 379-384.

\footnotetext{
${ }^{a}$ Authors who contributed equally to this article.

*Corresponding author: Zhi-yuan Zhang

Address: Shanghai Research Institute of Stomatology, Shanghai Ninth People's Hospital affiliated to Shanghai Jiao Tong University School of Medicine, Shanghai 200011, China

Tel: $862123271699 \quad$ Tax: $862163136856 \quad$ E-mail: zhzhy@omschina.org.cn
} 